\title{
PSA SECRETION AND CELL PROLIFERATION ARE AFFECTED BY NCOR1 SILENCING IN PROSTATE CANCER CELLS
}

\author{
Bruna Pasqualotto Costa1, Ilma Simoni Brum², \\ Lolita Schneider Pizzolato ${ }^{2}$, Vanderlei Biolchi ${ }^{3}$, Gisele Branchini ${ }^{1}$
}

\begin{abstract}
Introduction: The androgen receptor (AR) plays an important role in normal development of the prostate gland, as well as in prostatic neoplasms. Transcriptional regulation by AR is modulated by its interaction with co-activators or co-repressors, such as NCoR1 (nuclear receptor co-repressor 1 ), which is involved in reducing AR activity over the target gene transcription.
\end{abstract}

Methods: To identify the role of NCoR1 in the prostate cancer androgen independence in a cell line model, we aimed to evaluate the effects of silencing NCoR1 on prostate-specific antigen ( $P S A$ ) gene expression, the proliferative response and PSA secretion on the supernatant of C4-2B and LNCaP cells that were submitted to small interfering RNAs (siRNAs) transfection, and to treatments with different androgen dosages.

Results: In LNCaP and C4-2B cells with no dihydrotestosterone (DHT) treatment, a decrease in PSA mRNA expression was observed 48 hours and 72 hours after gene silencing in the siNCoR group when compared to the control and siNC groups. The $\mathrm{LNCaP}$ and $\mathrm{C} 4-2 \mathrm{~B}$ cells showed a biphasic pattern in response to dihydrotestosterone treatment in transfected groups ( $\mathrm{siNCoR}$ and siNC) as well as in the control condition (without transfection). The secretion of PSA in cell supernatant of LNCaP and C4-2B cells was higher in the siNCoR group, and, in relation to hormonal treatment, higher in the $10^{-8} \mathrm{M}$ DHT group.

Conclusions: A reduction in the NCoR1 levels seems to have a double influence on the activity of AR in PCa cells. These results suggest that NCoR may act as an AR co-repressor depending upon hormonal stimulation.

Keywords: Prostatic neoplasms; castration-resistant; prostatic neoplasm; NCoR1; gene silencing

Diseases that affect the prostate, such as chronic inflammatory lesions (prostatitis), benign prostatic hyperplasia (BPH), and prostate cancer (PCa) are related to the aging male ${ }^{1}$. Currently, $\mathrm{PCa}$ has the second highest worldwide incidence of cancer and is considered the sixth leading cause of death among men $^{2}$. The higher incidence of prostate cancer has increasingly motivated studies to investigate the mechanisms involved in the pathophysiology of this disease ${ }^{3}$.

Androgens play a critical role in the normal development of the prostate gland $^{1}$ and are also involved in the tumorogenesis of this organ ${ }^{4-6}$. Androgen receptor (AR) mediates the biological effects of androgens on cells $\mathbf{s}^{7,8}$, promoting epithelial luminal cell differentiation and regulating the transcription of the genes encoding proteins involved in the normal physiology of the prostate gland, like PSA ${ }^{3}$. Inhibition of the transcriptional activity of AR by androgen deprivation is still the primary choice treatment ${ }^{9}$. Initially, this approach is effective, but in about $70-80 \%$ of men, at a later stage the PCa cells acquire proliferative ability independent of androgen ${ }^{10}$. The efficacy of different therapeutic approaches to androgen suppression is directly related to the ability to consistently reduce serum total testosterone, but
Clin Biomed Res. 2020;40(1):37-43

1 Programa de Pós-Graduação em Patologia, Universidade Federal de Ciências da Saúde de Porto Alegre (UFCSPA). Porto Alegre, Rio Grande do Sul, Brasil.

2 Departamento de Fisiologia, Instituto de Ciências Básicas da Saúde, Universidade Federal do Rio Grande do Sul (UFRGS). Porto Alegre, Rio Grande do Sul, Brasil.

3 Centro de Ciências Biológicas e da Saúde, Universidade do Vale do Taquari (Univates). Lajeado, Rio Grande do Sul, Brasil.

Corresponding author: Gisele Branchini giseleb@ufcspa.edu.br Universidade Federal de Ciências da Saúde de Porto Alegre (UFCSPA)

Rua Sarmento Leite, 245. 90050-170, Porto Alegre, RS, Brasil. 
when progression occurs despite the maintenance of low serum levels of androgens, the cancer is then characterized as "androgen-independent" or "hormone-refractory"11.

Although the main components of the transcriptional pathway mediated by AR and the effect of androgens on it are described in detail, little is known about the role of co-activators and co-repressors during progression to androgen independence in the refractory $\mathrm{PC}$ a to hormonal deprivation ${ }^{12}$. Co-activators and co-repressors regulate the transcriptional activity of $A R$ by modulating the interactions between the receptor and the transcription initiation complex ${ }^{13}$; the co-activators are usually recruited to enhance gene expression while co-repressors bind to nuclear receptors mainly repressing transcription ${ }^{14}$. This increases the regulation by co-activators, as the low co-repressor expression has been shown to contribute to tumor progression ${ }^{15}$. The NCoR (nuclear receptor co-repressor) interacts with histone deacetylases to repress transcription ${ }^{16}$, so the NCoR interacts with AR and represses the transcriptional activity stimulated by dihydrotestosterone (DHT), indicating that this protein is a physiological regulator of AR activity ${ }^{17}$. The uncoupling of co-repressors and AR co-activator recruitment occur after the conformational changes promoted by the binding of androgens to the ligand binding site (LDB), so that the regulation exerted by these cofactors is dependent on the presence or absence of androgens in the prostatic cell ${ }^{18,19}$.

Studies involving silencing of the AR signaling pathway using RNAi can contribute to the understanding of the growth mechanisms of the tumor refractory to hormone deprivation therapy and to the development of new therapeutic approaches.

\section{METHODS}

\section{Cell Lines and Culture}

The prostatic cancer cell lines LNCaP $\left(\right.$ ATCC $^{\circledR}$, CRL-1740 ${ }^{\mathrm{TM}}$, passages number \#34 to \#38) and C4-2B (MD Anderson Cancer Center, passages number \#17 to \#20) were cultured in RPMI 1640 (GIBCO, BRL) supplemented with $10 \%(\mathrm{v} / \mathrm{v})$ fetal bovine serum (FBS) (GIBCO, BRL) and $1 \%(\mathrm{v} / \mathrm{v})$ kanamycin (Invitrogen, EUA). All cell lines were grown in a $37^{\circ} \mathrm{C}$, humidified incubator in the presence of $5 \%$ (v/v) $\mathrm{CO}_{2}$ gas. Passages were made with Trypsin/ EDTA $0.25 \%$ (GIBCO, BRL).

\section{Transfection with siRNA}

After LNCaP and C4-2B cells reached about 30 to $50 \%$ confluence, the culture medium was replaced by medium without antibiotics for 4 hours and then transfected using the Lipofectamine ${ }^{\circledR}$ 2000 reagent (Invitrogen, EUA). We previously tested three sequences of small interfering RNAs (siRNAs) (10nM) (HSS114352, HSS114353, HSS114354), separately and in combination, in order to choose the best conditions for gene silencing. The smallest NCoR1 mRNA expression was achieved with the combination of HSS114352 and HSS114353 sequences. The negative control group (siNC) received a scrambled sequence of siRNAs in the same concentration of NCoR1 siRNA (Stealth RNAi siRNA Negative Control Low GC and High GC, Invitrogen, EUA). To control the transfection efficiency, we used BLOCK-iT ${ }^{\mathrm{TM}}$ Fluorescent Oligo (Invitrogen, EUA), following the manufacturer's protocol.

\section{PSA gene expression}

The gene expression of PSA was assessed using Reverse Transcription Polymerase Chain Reaction (RT-PCR). LNCaP and C4-2B cells were seeded in 6 -well plates $\left(1 \times 10^{4}\right.$ cells $/$ well $)$, divided into three groups for analysis (Control, siNCoR and siNC). After 48 hours or 72 hours of transfection, cells were lyzed with Tryzol ${ }^{\circ}$ (Invitrogen, EUA) and the total RNA was extracted following the manufacturer's protocol. The RNA amount was quantified using the Qubit fluorimeter (Qubit ${ }^{\circledR}$ RNAAssay Kit, Invitrogen, EUA). Complementary DNA (cDNA) synthesis was performed using the Super Script ${ }^{\circledR}$ III First-Strand Synthesis System for RT-PCR (Invitrogen, EUA) from $1 \mu \mathrm{g}$ of RNA using the Oligo $\mathrm{dT}_{12-18}$ primer.

The evaluation of PSA gene expression was performed by RT-PCR of the PSA mRNA amplification with specific oligonucleotides ${ }^{20}$. The reaction was performed using Platinum SYBR $®$ Green qPCR SuperMix-UDG (Invitrogen, EUA) and in the 7500 Fast equipment (Applied Biosystems). The amplification reaction was normalized by the reference gene SdhA (succinate dehydrogenase complex, subunit A, flavoprotein (Fp)).

\section{Cell proliferation}

LNCaP and C4-2B cells were seeded in 24-well plates $\left(5 \times 10^{3}\right.$ cells/well), divided into three groups for analysis (Control, siNCoR and siNC) and separated according to different doses of dihydrotestosterone $\left(10^{-8} \mathrm{M} \mathrm{DHT}\right.$ and $\left.10^{-13} \mathrm{M} \mathrm{DHT}\right)$. After 48 hours of treatment, cells were transfected according to the analysis group. In the next 96 hours, the assessment of cell proliferation was performed using modified Sulforhodamine B assay (SR-B) ${ }^{21}$. For the SR-B assay, the culture medium was replaced by a culture medium without fetal bovine serum ${ }^{22}$ and the cells were fixed with $50 \%$ Trichloroacetic acid (TCA) $(100 \mu \mathrm{L}$ per well). 
The optical density (OD) was read at $510 \mathrm{~nm}$ on a spectrophotometer (AnthosZenyth $200 \mathrm{rt}$ ).

To obtain the proportion of cell growth of each experimental subgroup, the final absorbance values were adjusted relative to the initial number of cells plated $^{23}$. The control group was the subgroup without hormonal treatment, and which was not silenced, whose proliferation was considered to be $100 \%$.

\section{PSA secretion}

LNCaP and C4-2B cells were seeded in 24-well plates $\left(5 \times 10^{3}\right.$ cells/well). The secreted PSA dosage was measured in the cell supernatant at two different times: just before the transfection and 72 hours after the transfection. The dosage was assessed using the kit Cobas $®$ Total PSA (tPSA) (Roche Diagnostics, EUA). The results are expressed as delta PSA (PSA after transfection - PSA before transfection)/ proliferation rate.

\section{Statistical analysis}

Data from cell proliferation, PSA gene expression, and PSA secretion, were evaluated quantitatively by the Generalized Estimating Equations (GEE). The post-hoc Bonferroni test was performed to identify which groups differed. All analyses were performed using SPSS 18.0. Statistical significance was established at $p<0.05$.

\section{RESULTS}

\section{PSA mRNA expression}

In LNCaP cells, we observed a decrease of $P S A$ mRNA expression after 48 hours in the siNCoR group when compared to siNC $(p<0.05)$. All other groups showed no statistical difference in PSA expression, both at 48 and 72 hours after transfection $(\mathrm{N}=4)$ (Figure 1A).

In C4-2B cells, after 48 hours of transfection, the $P S A$ mRNA expression was lower in the siNCoR group compared to control and siNC groups $(p<0.05$, $\mathrm{N}=4$ ). After 72 hours, PSA mRNA expression was lower in the siNCoR group compared to the control group $(p<0.05, \mathrm{~N}=4)$ (Figure 1B).

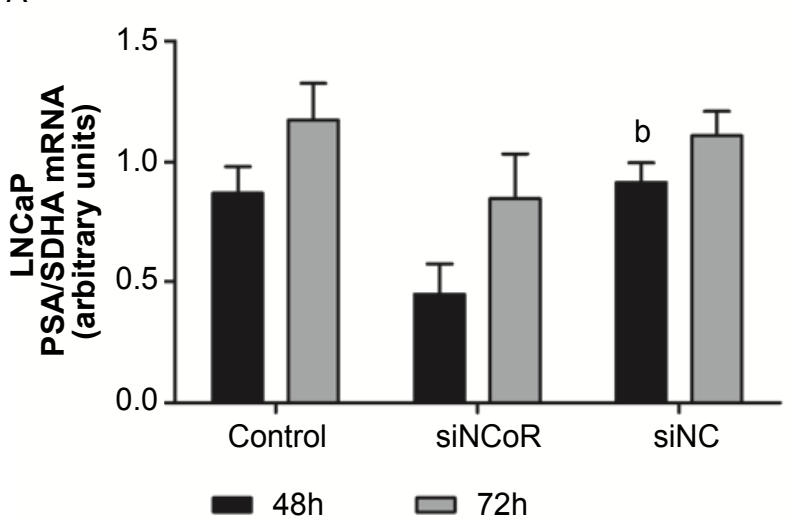

B

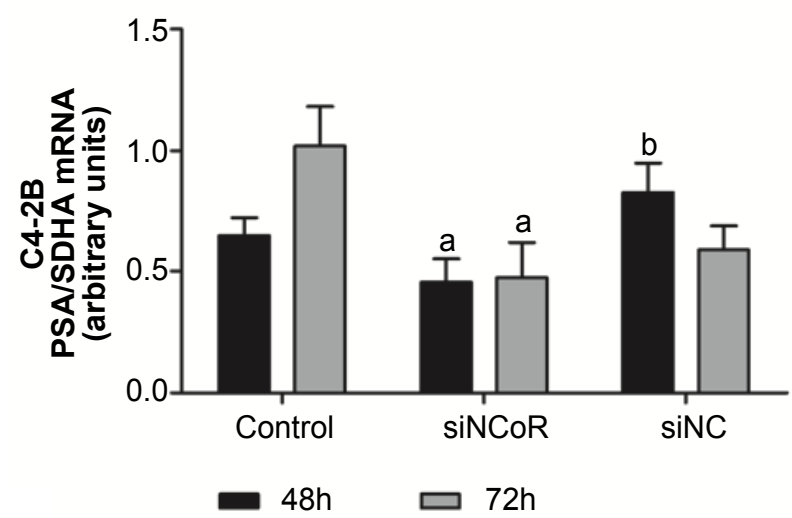

Figure 1: A: PSA mRNA expression in LNCaP; B: C4-2B cells (48 and 72 hours) after siRNA transfection. In the comparison between gene silencing groups (Control, siNCoR, siNC): "a" differs significantly from Control and " $b$ " differs significantly from siNCoR. The bars represent the mean \pm standard deviation. $p<0.05 ; n=4$.

\section{Cell Proliferation}

A biphasic pattern of response to DHT concentrations was observed in all groups (control, siNCoR, and siNC) in both cell lines. In the LNCaP cell line, cells treated with $10^{-13} \mathrm{M}$ DHT showed the highest levels of proliferation, followed by cells that were treated with $10^{-8} \mathrm{M} \mathrm{DHT}$ compared to cells without DHT (Control 5\%). When comparing the silencing groups, the siNC group showed a higher proliferation rate than siNCoR with the same $\mathrm{DHT}$ treatment (Figure 2A).

In the $\mathrm{C} 4-2 \mathrm{~B}$ cell line, treatment with $10^{-13} \mathrm{M}$ DHT promoted higher levels of cell proliferation; however, cells treated with $10^{-8} \mathrm{M}$ DHT showed less proliferation than the control group. The cells of the control group had proliferated more than siNCoR and siNC with the same DHT treatment (Figure 2B). 
A

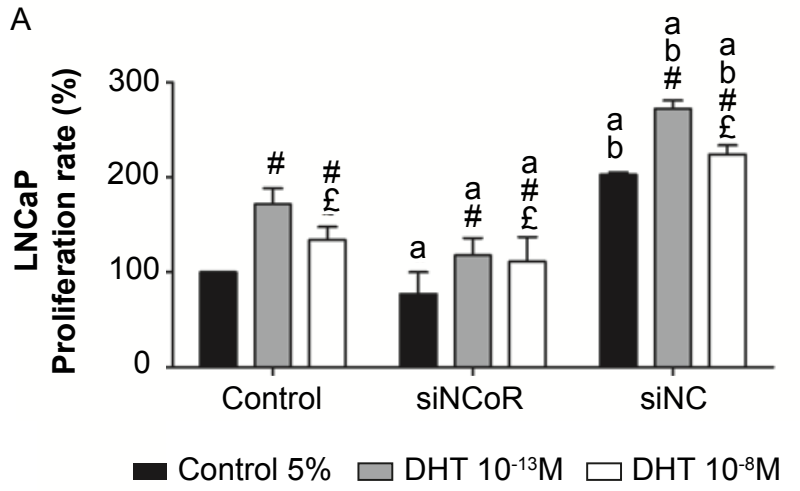

B

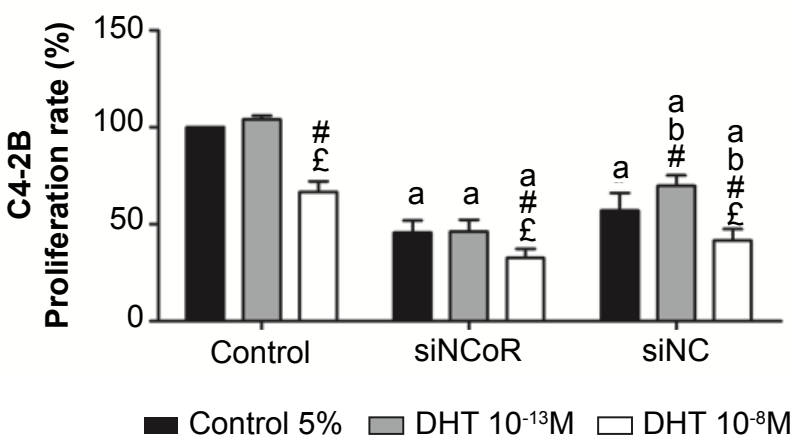

Figure 2: A: Proliferation rates of LNCaP; B: C4-2B cells submitted to NCoR1 silencing and DHT treatments. In the comparison between gene silencing groups (Control, siNCoR, siNC): "a" differs significantly from Control and " $b$ " differs significantly from siNCoR. In the comparison between hormonal treatments (Control 5\%, DHT 10-13 M, DHT 10-8 M): "\#" differs significantly from Control $5 \%$ and " $£$ " differs significantly from DHT $10^{-13} \mathrm{M}$. The bars represent the mean \pm standard deviation $p<0.05 ; n=4$.

\section{PSA secretion}

As observed in proliferation rates in response to hormonal treatment, PSA levels in the supernatant showed a biphasic pattern of response to DHT concentrations in all groups (control, siNCoR, and siNC). In both cell lines, cells treated with $10^{-8} \mathrm{M}$

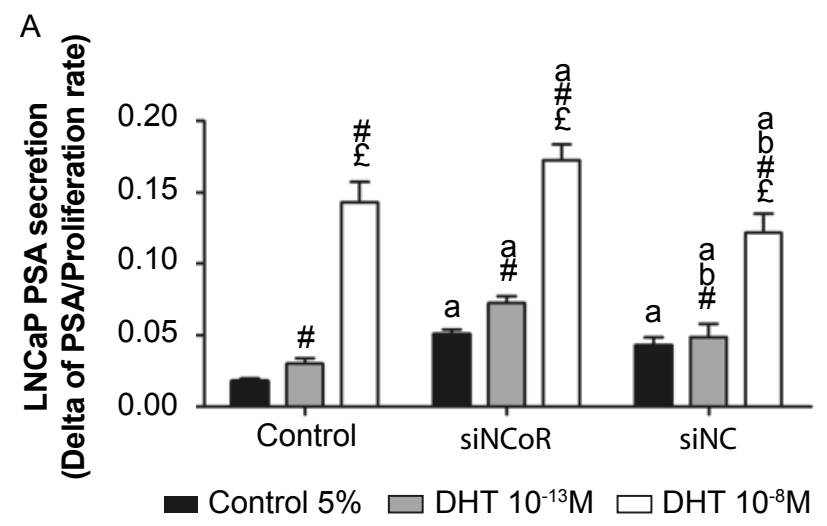

DHT presented the highest levels of PSA compared to cells treated with $10^{-13} \mathrm{M} \mathrm{DHT}$ and cells with no DHT treatment (Control 5\%) (Figure 3A and 3B). Cells treated with $10^{-13} \mathrm{M}$ DHT presented higher levels of PSA than cells without DHT treatment (Control 5\%).

Figure 3: A: PSA secretion levels in LNCaP; B: C4-2B cells submitted to NCoR1 silencing and DHT treatments. In the comparison between gene silencing groups (Control, siNCoR, siNC): "a" differs significantly from Control and " $b$ " differs significantly from siNCoR. In the comparison between hormonal treatments (Control 5\%, DHT 10-13 M, DHT 10-8 M): "\#" differs significantly from Control $5 \%$ and " $£$ " differs significantly from DHT $10^{-13} \mathrm{M}$. The bars represent the mean \pm standard deviation. $p<0.05 ; n=4$.

Comparing the silencing groups, we observed higher levels of PSA in the supernatant in the siNCoR group compared to control and siNC groups in almost all hormonal treatment subgroups.

\section{DISCUSSION}

The nuclear receptor co-repressor 1 is described as a transcription factor that represses transcriptional activity

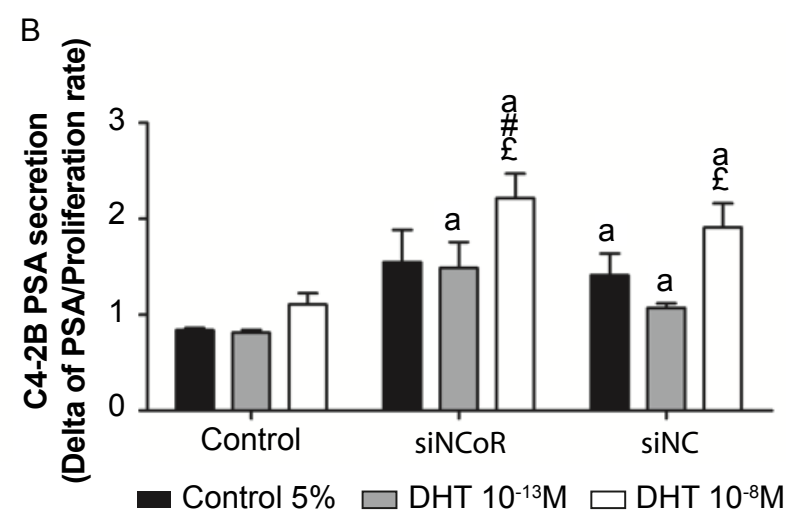

of the androgen receptor. When silencing NCoR1, it is assumed that the transcriptional repression mediated by the interaction of this co-repressor with AR would be reduced, which would result in greater activation of the AR signaling pathway. Then, the transcription of ARregulated genes, such as $P S A$, would increase, and the proliferation process could also be up-regulated in prostate cells in response to hormonal treatment. However, in our study, the reduced levels of NCoR1 in prostate cancer cells seemed to have no influence on 
the increased transcriptional androgen receptor activity in prostate cancer cells. When we evaluated the PSA mRNA expression at 48 hours of siRNA transfection, we observed lower levels of this transcript in both cell lines (LNCaP and C4-2B cells). Since there was no difference in gene expression between the control and SINC groups in this experimental condition, we suggest that this reduction in PSA gene expression took place due to the decrease in NCoR1 levels in these cells.

Hodgson and colleagues identified an increase in PSA expression after the depletion of $\mathrm{NCoR} 1^{9}$. The unexpected pattern observed in our study was also observed by Laschak and colleagues ${ }^{19}$, who evaluated the response of PC-3 cells (AR-negative prostate cancer cell line) and COS-1 cells (strain renal cell of monkey) to overexpression of the co-repressors NCoR and SMRT, co-transfected with AR and the PSA gene promoter. In COS-1 cells, the increased NCoR levels reduced the transcriptional activity of $A R$ by $50 \%$, as expected. PC-3 cells, however, had shown an increase in PSA expression. To test whether these results were specific to this cell line, since it has no endogenous AR, the LNCaP cell line was transfected with the luciferase reporter gene using the promoter of the probasin gene, which is androgen-responsive, to determine the transcriptional activity of $A R$ in the presence of increasing amounts of NCoR co-repressor. $\mathrm{LNCaP}$ cells overexpressing NCoR increased the transcriptional activity of endogenous AR by up to 2.7 times, depending on the amount transfected. Two other strains of prostate cancer cells were evaluated: DU-PCa and PCa-V (AR-positive), and the same pattern was found. Thus, increased levels of NCoR and SMRT were not able to suppress the transactivation of AR in LNCaP, DU-CaP, and V-CAP cells, nor in PC-3 cells. Instead, they promoted an increase in the transcriptional activity of $A R$ in $\mathrm{CaP}$ cells, suggesting a failure of NCoR to act as an AR, or even a new role for this protein in prostate cancer cells ${ }^{19}$. Our data agreed with the results from Laschak et al., since the NCoR did not respond as expected following overexpression of this co-repressor in prostate cancer cells ${ }^{19}$, nor when their levels were decreased in tumor cells, according to the data found in our study.

Proliferation rates in response to NCoR silencing combined with treatment with DHT were also evaluated. Generally, the siNCoR group presented lower proliferations rates compared to the control and siNC groups, in both cell lines. This pattern is also as unexpected as the transcriptional activity of $A R$, since we expected that activation of the AR signaling pathway could increase the proliferation of PCa cells. Since NCoR1 also acts to regulate the activity of nuclear receptors other than $A R$, it is possible that diminished levels can interfere with the activity of other nuclear receptors, thus changing the expression of other genes related to the rate of cell division.
Regarding the influence of dihydrotestosterone on proliferation rates, cells treated with $10^{-13} \mathrm{M} \mathrm{DHT}$ showed higher proliferation rates than cells without treatment and cells treated with $10^{-8} \mathrm{M} \mathrm{DHT}$. In LNCaP cells, which have a functional AR and are dependent on androgens, a biphasic pattern of response to DHT was observed: the group with the lower proliferation rate was the control group (without DHT), followed by the $10^{-8} \mathrm{M} \mathrm{DHT}$ and $10^{-13} \mathrm{M} \mathrm{DHT}$ groups, with the latter having the highest proliferation. These data corroborate previous studies, where the proliferation conditioned by androgens in response to different physiological doses is known to be biphasic: androgens in smaller doses at nanomolar levels $\left(10^{-9} \mathrm{M}\right)$ stimulate cell proliferation, while larger nanomolar level doses exert an anti-proliferative effect ${ }^{1,24}$. In C4-2B cells, we also observed responses to $10^{-13} \mathrm{M} \mathrm{DHT}$ and to $10^{-8} \mathrm{M} \mathrm{DHT}$, but the higher dose presented lower proliferation rates than the control group. This difference between these two cell types could be due to the response to androgens. Whereas $\mathrm{LNCaP}$ is dependent on the stimulus of androgens to proliferate ${ }^{25}, \mathrm{C} 4-2 \mathrm{~B}$ cells are androgen-sensitive cells, i.e., cells that can respond to androgen stimulation but do not depend on this stimulus for their maintenance ${ }^{26,27}$. Thus, the absence of DHT in the control group does not exert the same anti-proliferative effect in C4-2B cells as observed in LNCaP cells.

PSA secretion in supernatant of $\mathrm{C} 4-2 \mathrm{~B}$ and $\mathrm{LNCaP}$ presented a similar pattern, with the siNCoR and siNC groups presenting higher levels of this protein compared to the control group. Furthermore, the higher dose of DHT stimulated the highest secretion of this protein. Comparing the gene expression and secretion profiles, the results are opposite: cells submitted to the silencing of NCoR presented lower levels of PSA mRNA, but higher levels of PSA secretion. However, to evaluate the PSA mRNA expression, the cells were not submitted to hormonal treatment, and had received only the testosterone stimulation of fetal bovine serum, which may vary within nanomolar levels $\left(10^{-9} \mathrm{M}\right)^{28}$.

Lee and colleagues described that LNCaP cells accounted for variability of different $\mathrm{DHT}$ concentrations to which they are exposed. Low DHT concentrations $\left(10^{-12}\right.$ to $\left.10^{-10} \mathrm{M}\right)$ stimulated cell proliferation, but cells presented low levels of PSA secretion. In contrast, when exposed to high concentrations of DHT $\left(10^{-7} \mathrm{M}\right)$, cells reduced their proliferative capacity, accompanied by an increase in the production and secretion of PSA ${ }^{29}$. The response of $L N C a P$ cells to high concentrations of DHT is considered a result of a particular cellular event, since high levels of DHT can act by delaying AR degradation, which could culminate in the higher production of PSA and, in parallel, a decrease in cell proliferation ${ }^{29}$. Moreover, the treatment of $\mathrm{LNCaP}$ cells with androgens caused a decrease in the AR mRNA levels and a paradoxical increase in total AR quantity ${ }^{30,31}$. In addition, regarding the $\mathrm{PCa}$ cells dependence of 
androgens, there are differences in the co-repressors expression, as noticed by Villagran et al. (2015), which observed an increase of NCoR1 expression in LNCaP cells in absence of androgens, whereas there was a decrease in the C4-2B cells ${ }^{32}$. The preformed multisubunit complexes may lack co-repressor(s) in the androgen-resistant PCa cells, which could provide for AR functions as a transcription factor in the low circulating androgen levels ${ }^{32}$. Thus, the response of the distinct cell lines may be due differential set of co-activators/ co-repressors and their interactions to the transcription machinery in the PCa cells.

In our study, the evaluation of gene expression and PSA secretion was performed under different hormonal stimulation. In the presence of unknown levels of testosterone (from FBS), a hormone with a lower affinity for AR than DHT, the transcription of the $P S A$ gene was decreased in the group silenced for $\mathrm{NCoR}$ and may suggest the failure of NCoR to act as a co-repressor, since its silencing had no positive impact on the transcription of the PSA gene. In the presence of DHT stimulation, and higher binding affinity to AR, lower levels of NCoR led to the increased secretion of this protein in the supernatant of cells in culture. The well-characterized genomic pathway of androgen action regulates PSA gene transcription. Besides this pathway, it is known that androgens can act by nongenomic signaling pathways that are characterized by rapid response, indicating the absence of transcription and the translation of genes that are responsive to androgens. In addition, it is possible that non-genomic activity of AR might influence its genomic activity and that of other nuclear receptors ${ }^{18}$. Thus, it is possible that the high levels of $\mathrm{DHT}\left(10^{-8} \mathrm{M}\right)$ could be stimulating non-genomic pathways for AR signaling, which interact with genomic pathways and culminate in the increased translation of PSA mRNA or even trigger a signaling stabilization of PSA mRNA by increasing the levels of this secreted protein.

The study of the role of co-regulators in prostate cancer cell lines, as well as in primary cultures, may help to understand the pathophysiology of prostate cancer and the influence of these AR modulators on the development and maintenance of this malignancy, especially in cancers refractory to androgen deprivation therapy. Although NCoR has been extensively studied, most of these studies investigated the influence of this co-repressor on AR transcriptional activity in non-prostate cell lines ${ }^{17,19,33}$. The results of this study show slight differences from classic data presented in the literature, and further studies are needed to complement the information on the role of NCoR in androgen-independent tumors.

Our in vitro results showed that silencing the NCoR1 co-repressor in prostate cancer cells may result in increased AR activity, since there were higher rates of PSA secretion when under the stimulus of high doses of dihydrotestosterone $\left(10^{-8} \mathrm{M}\right)$. However, the increased transcriptional activity of $A R$ is not displayed in terms of an increase of PSAmRNA expression under the stimulation conditions of testosterone present in only FBS with which the medium is supplemented. Decreased levels of NCoR, contrary to expectations, do not appear to provide a proliferative advantage to C4-2B cells LNCaP, although all groups presented the expected increase in proliferation response to the stimulus of low doses of DHT $\left(10^{-13} \mathrm{M}\right)$.

Regardless of the mechanism involved, AR signaling plays a critical role in the development and progression of prostate cancer. Increased AR activity is fundamental in the processes that lead to androgen independence in prostate cancers refractory to androgen ablation therapy, which is the biggest obstacle to the successful treatment and control of PCa. Understanding the mechanisms that lead to the activation of the AR signaling pathway is extremely important for the development of effective therapies for hormone-refractory cancers. Further studies are needed to elucidate the molecular mechanisms by which AR co-repressors can respond in such different ways, leading to the activation of specific genes involved in processes such as androgen independence.

\section{Acknowledgments}

This work was supported by Fundação de Amparo à Pesquisa do Estado do Rio Grande do Sul (FAPERGS) (Grant number 168112-1/2012) and Fundo de Incentivo à Pesquisa do Hospital de Clínicas de Porto Alegre (FIPE-HCPA) (Grant number 08-172).

\section{REFERENCES}

1. Chatterjee B. The role of the androgen receptor in the development of prostatic hyperplasia and prostate cancer. Mol Cell Biochem. 2003;253(1-2):89-101.

2. Ferlay J, Shin HR, Bray F, Forman D, Mathers C, Parkin DM. Estimates of worldwide burden of cancer in 2008: GLOBOCAN 2008. Int J Cancer. 2010;127(12):2893-917.
3. Green SM, Mostaghel EA, Nelson PS. Androgen action and metabolism in prostate cancer. Mol Cell Endocrinol. 2012;360(1-2):3-13.

4. Latil $A$, Bièche I, Vidaud D, Lidereau $\mathrm{R}$, Berthon $\mathrm{P}$, Cussenot $\mathrm{O}$, Vidaud $\mathrm{M}$. Evaluation of androgen, estrogen (ER alpha and ER beta), and progesterone receptor expression in human prostate cancer by real-time quantitative reverse transcription-polymerase chain reaction assays. Cancer Res. 2001;61(5):1919-26.

5. Grönberg H. Prostate cancer epidemiology. Lancet. 2003;361(9360):859-64.

6. Yang Q, Fung KM, Day WV, Kropp $\mathrm{BP}$, Lin HK. Androgen receptor signaling is required for androgen- 
sensitive human prostate cancer cell proliferation and survival. Cancer Cell Int. 2005;5:8.

7. Gobinet J, Poujol N, Sultan C. Molecular action of androgens. $\mathrm{Mol}$ Cell Endocrinol. 2002;198(1-2):15-24.

8. Balk SP, Knudsen KE. AR, the cell cycle, and prostate cancer. $\mathrm{NuCl}$ Recept Signal. 2008;6:e001.

9. Hodgson MC, Astapova I, Hollenberg AN, Balk SP. Activity of androgen receptor antagonist bicalutamide in prostate cancer cells is independent of NCoR and SMRT corepressors. Cancer Res. 2007;67(17):8388-95.

10. Dehm SM, Tindall DJ. Ligandindependent androgen receptor activity is activation function2-independent and resistant to antiandrogens in androgen refractory prostate cancer cells. J Biol Chem. 2006;281(38):27882-93.

11. Harris WP, Mostaghel EA, Nelson PS, Montgomery B. Androgen deprivation therapy: progress in understanding mechanisms of resistance and optimizing androgen depletion. Nat Clin Pract Urol. 2009;6(2):76-85.

12. Koochekpour S. Androgen receptor signaling and mutations in prostate cancer. Asian J Androl. 2010;12(5):639-57.

13. Watson PJ, Fairall L, Schwabe JWR. Nuclear hormone receptor co-repressors: structure and function. Mol Cell Endocrinol. 2012;348-135(23):440-9.

14. Wang $Y$, Zhang $H$, Chen $Y$, Sun $Y$, Yang F, Yu W, et al. LSD1 is a subunit of the NuRD complex and targets the metastasis programs in breast cancer. Cell. 2009;138(4):660-72.

15. Chmelar R, Buchanan G, Need EF, Tilley W, Greenberg NM. Androgen receptor coregulators and their involvement in the development and progression of prostate cancer. Int $\mathrm{J}$ Cancer. 2007;120(4):719-33.

16. Cano P, Godoy A, Escamilla R, Dhir $\mathrm{R}$, Onate SA. Stromal-epithelial cell interactions and androgen receptor- coregulator recruitment is altered in the tissue microenvironment of prostate cancer. Cancer Res. 2007;67(2):511-9.

17. Cheng S, Brzostek S, Lee SR, Hollenberg AN, Balk SP. Inhibition of the dihydrotestosterone-activated androgen receptor by nuclear receptor corepressor. Mol Endocrinol. 2002;16(7):1492-501.

18. Bennett NC, Gardiner RA, Hooper JD, Johnson DW, Gobe GC. Molecular cell biology of androgen receptor signalling. Int J Biochem Cell Biol. 2010;42(6):813-27.

19. Laschak M, Bechtel M, Spindler KD, Hessenauer A. Inability of NCoR/ SMRT to repress androgen receptor transcriptional activity in prostate cancer cell lines. Int $\mathrm{J}$ Mol Med. 2011;28(4):645-51.

20. Souza AF, Brum IS, Neto BS, Berger $M$, Branchini G. Reference gene for primary culture of prostate cancer cells. Mol Biol Rep. 2013;40(4):2955-62.

21. Skehan $P$, Storeng $R$, Scudiero $D$, Monks A, McMahon J, Vistica D, et al. New colorimetric cytotoxicity assay for anticancer-drug screening. $J$ Natl Cancer Inst. 1990;82(13):1107-12.

22. Papazisis KT, Geromichalos GD, Dimitriadis KA, Kortsaris AH. Optimization of the sulforhodamine B colorimetric assay. J Immunol Methods. 1997;208(2):151-8.

23. Vichai V, Kirtikara K. Sulforhodamine B colorimetric assay for cytotoxicity screening. Nat Protoc. 2006;1(3):1112-6.

24. Brum IS, Morsch DM, Pozzobon A, Boeri VA, Geib G, Spritzer PM. Androgen-dependent expression of c-jun and c-fos in human nontransformed epithelial prostatic cells: association with cell proliferation. Horm Res. 2003;60(5):209-14.

25. Bebermeier JH, Brooks JD, DePrimo SE, Werner R, Deppe U, Demeter $\mathrm{J}$, et al. Cell-line and tissue-specific signatures of androgen receptorcoregulator transcription. J Mol Med (Berl). 2006;84(11):919-31.
26. Sramkoski RM, Pretlow TG, Giaconia JM, Pretlow TP, Schwartz S, Sy MS, et al. A new human prostate carcinoma cell line, 22Rv1. In Vitro Cell Dev Biol Anim. 1999;35(7):403-9.

27. Zhang Y, Castaneda S, Dumble M, Wang M, Mileski M, Qu Z, et al. Reduced expression of the androgen receptor by third generation of antisense shows antitumor activity in models of prostate cancer. Mol Cancer Ther. 2011;10(12):2309-19.

28. Oertel B. T Lindl und J. Bauer, Zell und Gewebekultur: Einführung in die Grundlagen sowie ausgewählte Methoden und Anwendungen. J Basic Microbiol. 1987;27(7):399-400.

29. Lee $C$, Sutkowski DM, Sensibars JA, Kim I, Amsel I, Shaw N, et al. Regulation of proliferation and production of prostate-specific antigen in androgen-sensitive prostatic cancer cells, LNCaP, by dihydrotestosterone. Endocrinology. 1995;136(2):796-803.

30. Krongrad A, Wilson CM, Wilson JD, Allman DR, McPhaul MJ. Androgen increases androgen receptor protein while decreasing receptor mRNA in LNCaP cells. Mol Cell Endocrinol. 1991;76(1-3):79-88.

31. Mizokami A, Saiga H, Matsui T, Mita T, Sugita A. Regulation of androgen receptor by androgen and epidermal growth factor in a human prostatic cancer cell line, LNCaP. Endocrinol Jpn. 1992;39(3):235-43.

32. Villagran MA, Gutierrez-Castro FA, Pantoja DF, Alarcon JC, Fariña MA, Amigo RF, et al. Bone stroma-derived cells change coregulators recruitment to androgen receptor and decrease cell proliferation in androgen-sensitive and castration-resistant prostate cancer cells. Biochem Biophys Res Commun. 2015;467(4):1039-45.

33. Agoulnik IU, Krause WC, Bingman WE, Rahman HT, Amrikachi M, Ayala GE, Weigel NL. Repressors of androgen and progesterone receptor action. $J$ Biol Chem. 2003;278(33):31136-48. 